\title{
EXTENSIONS OF PROXIMITY FUNCTIONS
}

\author{
DON A. MATTSON
}

\begin{abstract}
Let $P^{*}(X)$ be the algebra of bounded, real-valued proximity functions on a proximity space $(X, \delta)$, where $X$ is a dense subspace of a topological space $T$. In this paper we obtain several conditions which are equivalent to the following property: every member of $P^{*}(X)$ has a continuous extension to $T$. Examples concerning these results are included, one of which shows that this extension property is distinct from $C^{*}$-embedding.
\end{abstract}

1. Introduction. Let $\left(X, \delta_{1}\right)$ be a proximity space, where $X$ is a dense subspace of a topological space $T$, and let $P^{*}(X)$ be the algebra of bounded, real-valued proximity functions on $\left(X, \delta_{1}\right)$. In this paper, the following question is considered: when does every member of $P^{*}(X)$ have a continuous extension to $T$ ? Several conditions equivalent to this property are obtained.

For $T$ completely regular and Hausdorff, an example is provided to show that every member of $P^{*}(X)$ may have a continuous extension to $T$ when there is no compatible proximity relation $\delta$ for which $\left(X, \delta_{1}\right)$ is a $p$-subspace of $(T, \delta)$. It is also shown that the extension property characterized in the main results is distinct from $C^{*}$-embedding [3].

2. Preliminary results. By a uniform structure we shall mean a suitably defined collection of pseudometrics (see [3, Chapter 15]). The admissible, totally bounded uniform structure $\beta^{*}$ on $X$ generated by $P^{*}(X)$ is the unique totally bounded structure in the proximity class $\pi\left(\delta_{1}\right)$. (See [1] or [6].) Appropriate definitions and results concerning gauges on proximity spaces may be found in [4].

The theory of round filters is developed in [2] and [6].

Let $\delta_{R}$ be the proximity relation on the real numbers induced by the standard metric. Denote the negation of a proximity relation $\delta$ by $\bar{\delta}$.

LEMMA 1. If each point $x$ in $T$ is a cluster point of a unique maximal round filter $\mathcal{F}_{x}$ on $\left(X, \delta_{1}\right)$, then every gauge $\sigma \in \mathcal{\odot}^{*}$ has an extension to a pseudometric $\bar{\sigma}$ on $T$.

Received by the editors December 9, 1969.

AMS 1969 subject classifications. Primary 5430; Secondary 5453, 5460.

Key words and phrases. Proximity space, $p$-subspaces, algebras of bounded realvalued proximity functions, Smirnov compactification, $C^{*}$-embedding, round filters, gauges, continuous extension of functions, Stone-Čech compactification. 
Proof. Let $\delta_{1} X$ be the Smirnov compactification of $\left(X, \delta_{1}\right)$. Since $\rho^{*}$ is totally bounded, by Theorem 1 of [4], every gauge $\sigma \in \odot^{*}$ has a unique extension to a gauge $\sigma^{*}$ on $\delta_{1} X$. The collection $\left\{\sigma^{*}: \sigma \in \rho^{*}\right\}$ is the unique admissible uniform structure on $\delta_{1} X$ (see [3]). Now for each $x \in T$, let $x^{*}$ be the unique cluster point of $\mathcal{F}_{x}$ in $\delta_{1} X$. Define $\bar{\sigma}(x, y)=\sigma^{*}\left(x^{*}, y^{*}\right)$, for all $x, y \in T$. Evidently, $\bar{\sigma}$ is an extension of $\sigma$ to a pseudometric on $T$, and the proof is complete.

For $A \subseteq T$, denote $T-A$ by $c_{T} A$.

LEMMA 2. If each point $x$ in $T$ is a cluster point of a unique maximal round filter $\mathcal{F}_{x}$ on $\left(X, \delta_{1}\right)$, and if $\bar{\sigma}$ is the extension to $T$ of a gauge $\sigma \in \mathcal{P}^{*}$, then for each $x \in T$ and $\epsilon>0$, the $\epsilon$-ball about $x$ determined by $\bar{\sigma}$ contains a $T$-neighborhood of $x$.

Proof. Suppose for some $x \in T, \sigma \in \rho^{*}$ and $\epsilon>0$, every open $T$ neighborhood $N$ of $x$ satisfies $N \cap c_{T} N(\bar{\sigma}, \epsilon) \neq \varnothing$, where $N(\bar{\sigma}, \epsilon)$ is the $\epsilon$-ball about $x$ determined by $\bar{\sigma}$. For each $N$, choose $y_{N} \in N \cap c_{T} N(\bar{\sigma}, \epsilon)$, and let $\mathcal{F}_{N}$ be the maximal round filter on $\left(X, \delta_{1}\right)$ which clusters at $y_{N}$. Then there exists $F_{N} \in \mathcal{F}_{N}$, where $\sigma\left[F_{N}\right] \leqq \epsilon / 4$. Also, choose $E \in \mathcal{F}_{x}$ for which $\sigma[E] \leqq \epsilon / 4$. Then if $\sigma\left(E, F_{N}\right)<\epsilon / 4$, we have $\bar{\sigma}\left(x, y_{N}\right)$ $\leqq 3 \epsilon / 4$, which contradicts the choice of $y_{N}$. Set $A=\bigcup_{N} F_{N}$. Then $A \bar{\delta}_{1} E$, since $\sigma$ is a gauge.

The collection of sets $N \cap A$ is a base for a filter on $X$. Let $\varepsilon_{1}$ denote the round hull of this filter, and let $\mathcal{E}$ be a maximal round filter on $\left(X, \delta_{1}\right)$ containing $\mathcal{E}_{1}$. Then $\mathcal{E} \neq \mathfrak{F}_{x}$ and by the uniqueness of $\mathcal{F}_{x}, x$ is not a cluster point of $\mathcal{E}$. Hence, there exist an open $T$-neighborhood $N_{1}$ of $x$ and $E_{1} \in \mathcal{E}$ for which $N_{1} \cap E_{1}=\varnothing$. Take $E_{2} \in \mathcal{E}$, where $E_{2} \ll E_{1}$, so that $\left(N_{1} \cap A\right) \ll\left(X-E_{2}\right)$. Then $E_{2}, X-E_{2} \in \mathcal{E}$, which is impossible. This completes the proof.

3. Extensions of mappings. We now prove the main theorem on extensions.

Theorem. Let $\left(X, \delta_{1}\right)$ be a proximity space, where $X$ is a dense (topological) subspace of $T$. Then the following are equivalent:

(i) Every point $x$ in $T$ is a cluster point of a unique maximal round filter $\mathcal{F}_{x}$ on $\left(X, \delta_{1}\right)$

(ii) Every $\sigma \in \odot^{*}$ has a unique extension to a continuous pseudometric $\bar{\sigma}$ on $T$.

(iii) The canonical injection of $\left(X, \delta_{1}\right)$ into its Smirnov compactification $\delta_{1} X$ can be extended uniquely to a continuous mapping of $T$ into $\delta_{1} X$.

(iv) Every $f_{1}$ in $P^{*}(X)$ has an extension to a member $f$ of $C^{*}(T)$. 
(v) $\delta_{1}$ has a unique extension to a generalized proximity relation $\delta$ on $T$ such that $A \delta B$ for every pair of subsets $A, B$ with intersecting $T$ closures.

(vi) If $A \bar{\delta}_{1} B$ in $X$, then $\mathrm{Cl}_{T} A \cap \mathrm{Cl}_{T} B=\varnothing$.

Proof. (i) implies (ii). Lemma 1 provides the extension, and the continuity follows immediately from Lemma 2 , so that $\bar{\sigma}$ is the unique extension of $\sigma$ to $T$.

(ii) implies (iii). The collection $D=\left\{\bar{\sigma}: \sigma \in \rho^{*}\right\}$ is a totally bounded uniform structure on $T$ (possibly nonseparating and possibly nonadmissible). Let $T^{*}$ be the completion of the separated uniform space associated with $(T, D)$. Then there is a uniformly continuous mapping $J$ of $T$ into $T^{*}$, where $J[T]$ is dense in the compact, Hausdorff space $T^{*}$. Since $X$ is dense in $T$ and each $\bar{\sigma}$ agrees with $\sigma$ on $X, T^{*}$ must be the Smirnov compactification of $\left(X, \delta_{1}\right)$. Thus (iii) follows from the uniqueness of the Smirnov compactification.

(iii) implies (iv). Let $J$ be the continuous extension to $T$ of the canonical injection of $\left(X, \delta_{1}\right)$ into $\delta_{1} X$. If $f_{1} \in P^{*}(X)$, let $f_{1}^{*}$ be the unique extension of $f_{1}$ to a member of $C\left(\delta_{1} X\right)$. Define $f=f_{1}^{*} \circ \mathfrak{J}$. Evidently, $f$ is the unique continuous extension of $f_{1}$ to $T$.

(iv) implies (v). For subsets $A$ and $B$ of $T$, define $A \delta B$ if and only if no $f$ in $C^{*}(T)$ which is the extension of some $f_{1}$ in $P^{*}(X)$ separates $A$ and $B$. It is easily verified that $\delta$ is a generalized proximity (not necessarily compatible with the topology on $T$, e.g. example (A)), and that $\delta$ agrees with $\delta_{1}$ on $X$. If $\mathrm{Cl}_{T} A \cap \mathrm{Cl}_{T} B \neq \varnothing$, clearly $A \delta B$.

Now if $\delta^{*}$ is any generalized proximity relation on $T$ which satisfies (v), then the open sets in the $\delta^{*}$-topology are open in the $T$-topology. Then, since $X$ is dense in $T$, we have $\delta^{*}=\delta$, so that $\delta$ is unique.

(v) implies (vi). Trivial.

(vi) implies (i). For $x \in T$, define $\mathcal{F}_{x}$ on $\left(X, \delta_{1}\right)$ by $A \in \mathcal{F}_{x}$ if and only if there exists $B$ for which $B \ll A$ and $x \in \mathrm{Cl}_{T} B$. If $A_{1}, A_{2} \in \mathcal{F}_{x}$, choose $B_{1}^{*}$ and $B_{2}^{*}$ satisfying $B_{1} \ll B_{1}^{*} \ll A_{1}$ and $B_{2} \ll B_{2}^{*} \ll A_{2}$, where $x \in \mathrm{Cl}_{T} B_{1} \cap \mathrm{Cl}_{T} B_{2}$. Since $x \notin \mathrm{Cl}_{T}\left(X-B_{1}^{*}\right) \cup \mathrm{Cl}_{T}\left(X-B_{2}^{*}\right)$ by (vi), we have $x \in \mathrm{Cl}_{T}\left(B_{1}^{*} \cap B_{2}^{*}\right)$. Now $\left(B_{1}^{*} \cap B_{2}^{*}\right) \ll\left(A_{1} \cap A_{2}\right)$, so that $A_{1} \cap A_{2} \in F_{x}$. Clearly, $\mathcal{F}_{x}$ is closed under supersets, hence $\mathfrak{F}_{x}$ is a round filter on $\left(X, \delta_{1}\right)$ and $x$ is a cluster point of $\mathcal{F}_{x}$.

Now if $A \in F_{x}$ and $\left\{A_{k}: k=1, \cdots, n\right\}$ is any $p$-cover of $A$, since $x \in \mathrm{Cl}_{T} A$, the definitions of $\mathcal{F}_{x}$ and $p$-cover imply that some $A_{k} \in \mathcal{F}_{x}$. Thus, by Proposition 2 of [2], $\mathcal{F}_{x}$ is a maximal round filter.

Finally, let $\varepsilon$ be any maximal round filter on $\left(X, \delta_{1}\right)$, where $x$ is a cluster point of $\mathcal{E}$. If $\mathcal{F}_{x} \neq \mathcal{E}$, there exists $F \in \mathcal{F}_{x}$, where $F \notin \mathcal{E}$. Choose $F_{1}, F_{2} \in \mathcal{F}_{x}$ satisfying $F_{2} \ll F_{1} \ll F$. Now $\left\{F, X-F_{1}\right\}$ is a $p$-cover of 
$\left(X, \delta_{1}\right)$, so that $X-F_{1} \in \mathcal{E}$. But then $x \in \mathrm{Cl}_{T}\left(X-F_{1}\right) \cap \mathrm{Cl}_{T} F_{2}$, which contradicts (vi).

Thus $\mathcal{F}_{x}$ is unique, and the proof is complete.

If $T$ is a completely regular Hausdorff space, then $C^{*}(T)$ induces a compatible proximity relation $\beta$ for $T$ by defining $C \beta D$ in $T$ if and only if no member of $C^{*}(T)$ completely separates $C$ and $D$. Then the Stone-Cech compactification $\beta T$ of $T$ is the Smirnov compactification of $(T, \beta)$. Now the following result is immediate.

COROLlaRy. If $X$ is dense in $T$ and if $\delta_{1}$ is a proximity relation for $X$, then the following are equivalent:

(i) Every point of $T$ is a cluster point of a unique maximal round filter on $\left(X, \delta_{1}\right)$.

(ii) The canonical injection of $\left(X, \delta_{1}\right)$ into its Smirnov compactification $\delta_{1} X$ has an extension to a proximity mapping of $(T, \beta)$ into $\delta_{1} X$.

(iii) There is a continuous mapping of $\beta T$ onto $\delta_{1} X$ which leaves $X$ point-wise fixed.

(iv) Every member of $P^{*}(X)$ has an extension to a member of $C^{*}(T)$.

(v) $\beta \mid X \subseteq \delta_{1}$.

Proof. The equivalence of (ii) and (iii) follows immediately from the extension properties [3] of the Stone-Cech compactification.

ExAmples. (A) Let $X=(0,1)$ with $\delta_{1}=\delta_{R} \mid X$, and take $T=\beta X$, where $\beta X$ is the Stone-Čech compactification of $X$. Then $\beta X$ has a unique compatible proximity relation $\delta$ and $X$ is $C^{*}$-embedded in $\beta X$, so that every member of $P^{*}(X)$ has an extension to a member of $C(\beta X)=P^{*}(T)$. However, there are members of $C(\beta X)$ whose restrictions to $X$ are not in $P^{*}(X)$, so that $\delta \mid X$ is properly contained in $\delta_{1}$. Thus one, hence all, of the conditions of the corollary may hold even when there is no $\delta$ for which $\left(X, \delta_{1}\right)$ is a $p$-subspace of $(T, \delta)$.

(B) The conditions of the corollary may hold without $X$ being $C^{*}$-embedded in $T$. Let $X=[-1,0) \cup(0,1]$ and $T=[-1,1]$. Let $\delta$ be the (unique) proximity relation for $T$ and take $\delta_{1}=\delta \mid X$. Then (v) of the corollary holds, so that every member of $P^{*}(X)$ has an extension to a member of $P^{*}(T)=C^{*}(T)$, but clearly $X$ is not $C^{*}$-embedded in $T$.

(C) Let $N$ denote the positive integers with the discrete topology, and let $N^{*}$ be the one-point compactification of $N$. Let $\delta_{1}$ be the discrete proximity relation on $N$. If $\varepsilon_{1}$ is the round filter of all subsets of $N$ containing all but a finite number of even integers, and if $\varepsilon_{2}$ is the round filter consisting of all sets containing all but finitely many odd integers, and if $\mathcal{F}_{1}$ and $\mathcal{F}_{2}$ are maximal round filters containing $\mathcal{E}_{1}$ and $\mathcal{E}_{2}$, respectively, then both $\mathcal{F}_{1}$ and $\mathcal{F}_{2}$ converge to the ideal 
point of $N^{*}$. Thus (i) of the corollary fails. Since there is only one compatible proximity relation $\delta$ for $N^{*},\left(N, \delta_{1}\right)$ cannot be a $p$-subspace of $\left(N^{*}, \delta\right)$ by the corollary. In this case, $\delta_{1}$ is properly contained in $\delta \mid N$.

(D) In the theorem, "maximal round filter" cannot be replaced by "cluster" (see [5]). Let $X$ be the graph of $y=\sin \left(x^{-1}\right)$ for $x$ in $(0,1]$, and take $T=X \cup\{(0,0)\}$, where the topologies in $X$ and $T$ are inherited from the plane. Define $A \delta_{1} B$ in $X$ if and only if $\mathrm{Cl}(p A) \cap \mathrm{Cl}(p B) \neq \varnothing$, where $p$ is the projection into the horizontal axis. Now the extension theorem holds for $X$ and $T$ (for example, apply (vi)), but $(0,0)$ is not a cluster point of any cluster in $\left(X, \delta_{1}\right)$.

To the referee goes the credit for (iii) and (v) of the theorem and for example (D). The author is also indebted to the referee for his valuable comments concerning the proofs, and for suggesting the inclusion of (ii) of the theorem.

\section{REFERENCES}

1. E. M. Alfsen and J. E. Fenstad, On the equivalence between proximity structures and totally bounded uniform structures, Math. Scand. 7 (1959), 353-360. MR 22 \#5958.

2. - A note on completion and compactification, Math. Scand. 8 (1960), 97104. MR 23 \#A3543.

3. L. Gillman and M. Jerison, Rings of continuous functions, The University Series in Higher Math., Van Nostrand, Princeton, N. J., 1960. MR 22 \#6994.

4. S. Leader, On completion of proximity spaces by local clusters, Fund. Math. 48 (1959/60), 201-216. MR 22 \#4047.

5. - On clusters in proximity spaces, Fund. Math. 47 (1959), 205-213. MR $22 \# 2978$.

6. W. Thron, Topological structures, Holt, Rinehart, and Winston, New York, 1966. MR 34 \#778.

Trinity College, Hartford, Connecticut 06106 